\title{
INFLUENCE OF THE SYMPATHETIC NERVOUS SYSTEM ON THE INTRA-OCULAR PRESSURE AND VASCULAR CIRCULATION OF THE EYE*†
}

\author{
BY
}

\author{
D. P. GREAVES AND E. S. PERKINS
}

\author{
From the Institute of Ophthalmology, London \\ Director of Research: Sir Stewart Duke-Elder
}

THE first section of the following paper describes the effects on the intraocular pressure of cutting and stimulating the cervical sympathetic nerves in cats and rabbits. This section is a repetition of previous workers' experiments, except that we have recorded simultaneously on the same film the intra-ocular pressure of both eyes and the blood pressure, thus enabling intra-ocular pressure changes resulting from general blood pressure effects to be distinguished from local sympathetic effects.

The subsequent sections deal with the appearance in vivo of the aqueous veins and choroidal circulation in rabbits. The changes observed during stimulation of the sympathetic nerve and the results of injecting dyes and Indian ink into the carotid artery and anterior chamber are described.

\section{Historical Survey}

It is now almost 50 years since Henderson and Starling published their classical paper on the effect on the intra-ocular pressure of stimulation of the sympathetic and third cranial nerves of cats. Höltzke (1883) and Adamük (1866) seem to have been the first workers to investigate adequately these effects and to describe the changes in the intra-ocular pressure on section of the cervical sympathetic. Since then many experiments have been performed by different workers.

A fall in the intra-ocular pressure on cutting the cervical sympathetic nerve was recorded by Höltzke (1883), Graser (1883), Adamük (1866), Lagrange and Pachon (1900), Schmidt-Rimpler (1900), and Stock (1910).

Leber (1903) noted a slight rise followed by a slower fall in the intra-ocular pressure after stimulating the sympathetic in dogs and cats. In rabbits the initial rise was absent. Similar results are reported by Henderson and Starling (1904), Lodato (1906), Wessely (1908), Rochat (1919), and Bourquin (1925). These discrepancies between species are no doubt dependent on the presence and amount of smooth muscle in the orbit.

Adler (1924) noticed a decrease in the formation of plasmoid aqueous following paracentesis during sympathetic stimulation in experimental animals. Thiel (1929) noted a short rise followed by a longer fall in both normal and glaucomatous human eyes; he also reported a bilateral effect on the intra-ocular pressure from stimulation of one sympathetic.

* Received for publication November 12, 1951.

† This work has been done with a grant from the Alexander Piggott Wehrner Memorial Trust Fund. 
Colle and Duke-Elder (1931) produced a rise in the intra-ocular pressure of exsanguinated dogs by stimulation of the sympathetic, thus demonstrating that the initial rise in normal animals is due to contraction of smooth muscle in the orbit.

Yata (1930) stated that sympathetic section had no effect on the normal intraocular pressure but stimulation of the nerve lowered both intra-ocular pressure and blood pressure. Vidal and Malbran (1944) reported two cases of unilateral stellectomy in man in which the intra-ocular pressure was found to be equal and normal in the two eyes, 2 and 4 years respectively after operation.

Jaffe (1948), working with cats, showed that section of the sympathetic caused a fall in the intra-ocular pressure in the ipsilateral eye, which returned to normal in $\mathbf{2}$ or $\mathbf{3}$ days. He thought this fall due to loss of tone in the smooth muscle of the orbit.

Schmerl and Steinberg (1949) found that stimulation of the superior cervical ganglion caused a lowering of the intra-ocular pressure from 7 to $11 \mathrm{~mm}$. $\mathrm{Hg}$ in rabbits. Davson and Matchett (1951) reported that section of the sympathetic in rabbits is sometimes followed by a large rise in the intra-ocular pressure which subsides within 30 to $60 \mathrm{~min}$. Stimulation of the cut peripheral end caused a fall in the intra-ocular pressure.

In the majority of the above experiments, simultaneous blood-pressure records have not been done, and the intra-ocular pressure has been measured by a Schiötz or other type of indirect tonometer. We have attempted to obtain more accurate measurements of changes in the intra-ocular pressure of both eyes and simultaneous blood-pressure records of the result of section and stimulation of the pre-ganglionic cervical sympathetic nerve.

Methods of measuring the intra-ocular pressure fall into two groups: direct measurement by the insertion of a needle into the anterior chamber, and tonometric methods. Many workers have relied on repeated readings of the tension with a Schiötz type tonometer, and while this may be a useful method in long-term experiments and when large pressure changes are being measured, it is not sufficiently reliable for small changes and has the additional disadvantage that repeated readings tend to massage fluid out of the eye and thus to reduce the intra-ocular pressure.

We have therefore used the method of direct measurement, which allows continuous recording over several hours, if necessary. Details of this technique were extensively discussed as early as 1868 by von Hippel and Grünhagen. To maintain accuracy, the main factors to consider are:

(1) External pressure on the globe on the insertion of the needle must be small ; for this purpose the needle must be sharp.

(2) There should be no loss of aqueous" on insertion of the needle.

(3) The volume displacement of the needle on insertion should be small ; in our own experiments it was never more than $1.2 \mathrm{ml}$. According to Wessely (1908), this increase of volume in the eye of a rabbit with a pressure of $20 \mathrm{~mm}$. $\mathrm{Hg}$ will result in an increase of that pressure by about $1 \mathrm{~mm}$. Hg.

(4) The movement of the rubber membrane of the manometer should likewise be small ; in our apparatus the volume change, as measured directly by a micrometer syringe, was only $0.018 \mathrm{ml}$. for movement from 20 to $50 \mathrm{~cm}$. saline, i.e., $0.6 \mu \mathrm{l}$. per $\mathrm{cm}$. saline, assuming the membrane to move in direct proportion to the volume change (which was practically the case). 


\section{Methods}

The animals were anaesthetized with nembutal, $1 \frac{1}{2}$ gr. per lb. bodyweight, intraperitoneally. The sympathetic nerves between the middle and superior cervical ganglion were dissected out on both sides of the neck. The right femoral artery was next isolated and cannulated, using a siliconed glass cannula of the usual design, the blood pressure being recorded by a manometer of similar design to that used for the intra-ocular pressure but with a heavier gauge rubber membrane.

The intra-ocular pressure was recorded by inserting into the anterior chamber a No. 12 hypodermic needle connected by narrow polythene tubing to a manometer of the type described by Davson and Purvis (1950). Pantocaine 2 per cent. was instilled into the eyes before inserting the needles, as a rapid rise of intra-ocular pressure was seen to follow in a few cases if no local anaesthetic was used. The manometer system was filled with normal saline, and at the moment of insertion of the needle a reservoir connected to the manometer was adjusted to give a head pressure of $20 \mathrm{~cm}$. saline- the tap being closed again as soon as the needle was in place.

The movement of beams of light reflected from the mirrors of the manometers was recorded on a moving film in a specially designed camera. See Figs 1 and 2.

When the intra-ocular pressure had reached a steady level in each eye, one sympathetic nerve was cut. The second sympathetic was left intact in some experiments and cut after an interval of about $10 \mathrm{~min}$. in others. Stimulation was effected with an electronic square-wave generator (Attree, 1950 : oscillator circuit of Fig. 2, and pulse generator of Fig. 3) using 4 volts, 1 millisecond pulse width, and 50-cycle frequency. The duration of the stimulus is shown on the tracings.

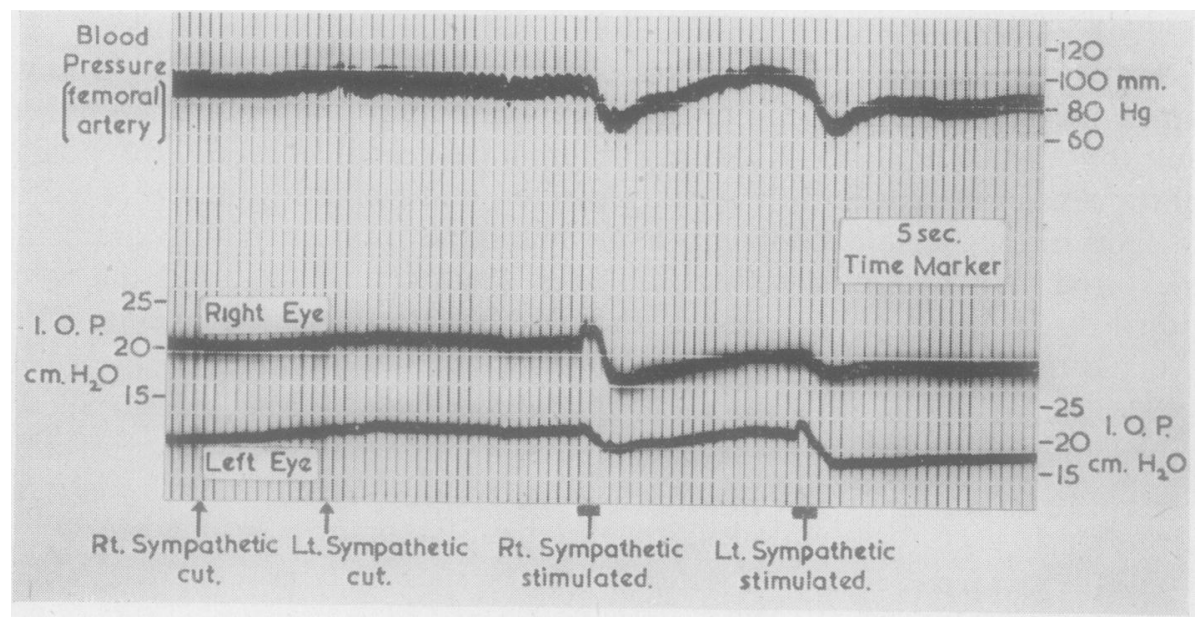

FIG. 1.- Section and stimulation of cervical sympathetic nerve in Cat 40.

Simultaneous record of the blood pressure in the femoral artery and of the intraocular pressure of each eye. The broken vertical lines are spaced at intervals of $5 \mathrm{sec}$. The immediate short rise of the ipsilateral intra-ocular pressure on stimulation is muscular in origin. Thereafter there is a fall in both eyes on unilateral stimulation of the sympathetic, due partly to the fall in general blood pressure and partly to local events in the eye. The difference between the fall in the ipsilateral and contralateral eye indicates the change due to local events. 


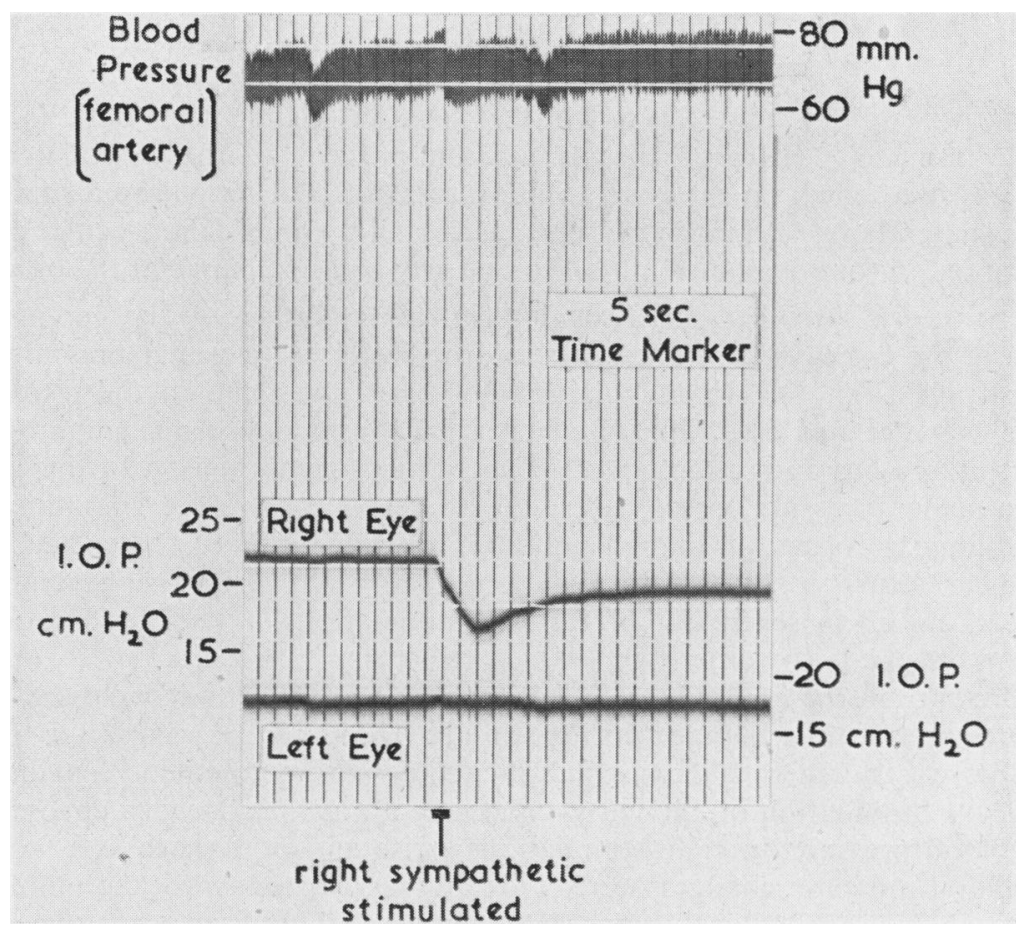

Fig. 2.-Stimulation of cervical sympathetic nerve in the rabbit.

Simultaneous record of the blood pressure in the femoral artery and of the intraocular pressure of each eye. There is no change in the blood pressure, and a fall in the ipsilateral eye is due solely to changes in the intra-ocular circulation.

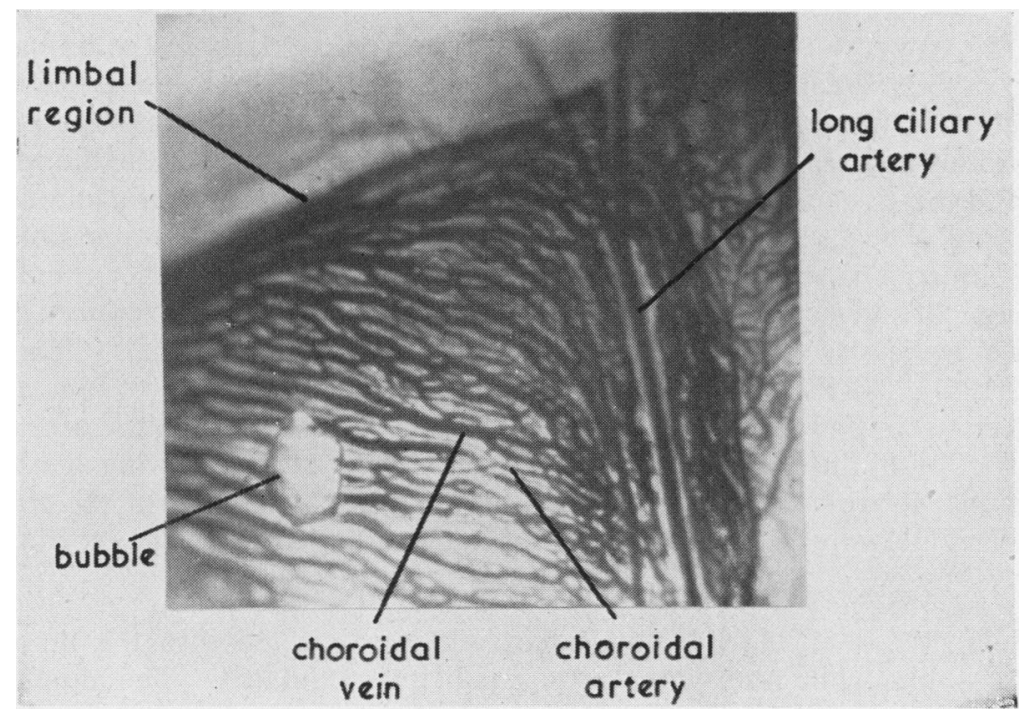

Fig. 3.-Photograph of choroidal vessels near limbus, together with a long posterior ciliary artery seen through the cleared sclera after injection of Indian ink into the carotid artery. The blob is an artefact due to a bubble in the oil. 


\section{Results}

PART I

(a) Section of Cervical Sympathetic.-Section of the sympathetic in the cat has little immediate effect on the intra-ocular pressure of the contralateral eye, and no constant effect on the general blood pressure, but may have a small effect on the ipsilateral eye. In nine experiments, a small rise in the intra-ocular pressure was recorded in four eyes, a small fall in one eye, and no change in the other four.

(b) Stimulation of Either Sympathetic.-Stimulation of the cervical sympathetic in the cat caused in every case a drop in the intra-ocular pressure in the ipsilateral eye (Fig. 1). In the majority of cases the drop in the intra-ocular pressure was preceded by a rapid rise caused by contraction of the smooth muscle in the orbit and lids and retraction of the nictitating membrane. A further drop in the intra-ocular pressure, presumably due to relaxation of the smooth muscle, occurred on many occasions immediately after the stimulation ceased, to be followed by a slower return to the pre-stimulation level. A fall in the general blood pressure occurred in seven out of thirteen stimulations, a rise in four, and no significant change in the other two.

The pressure in the contralateral eye was found to follow passively the general blood pressure; it thus changed during stimulation, as can be seen in the tracings. This effect was noted in all cases in which the right sympathetic had been cut previously. Stimulation of the left sympathetic always produced a change in the general blood pressure, in eight stimulations a rise and in three cases a fall.

The blood-pressure changes were probably associated with the number of depressor fibres in the cervical sympathetic nerve, a fall of blood pressure occurring when depressor fibres were included. In the absence of depressor fibres, a rise of blood pressure might occur reflexly due to reduction of blood flow to the head. In the rabbit, stimulation of the cervical sympathetic produced a fall in intra-ocular pressure, the initial rise seen in the cat being absent (Fig. 2).

PART II. AqueOUS VeINS.-These can be observed regularly in the limbal circulation of rabbits (Weekers and Prijot, 1950; Greaves and Perkins, 1951). We watched the effect of stimulating the cervical sympathetic on the appearance of these vessels.

If a stimulus of less than 5 volts is used, a decrease in calibre of the blood vessels is seen and there is an apparent increase in the flow of aqueous. If a stimulus of 5 volts is used, the whole limbal region becomes avascular and it is impossible to tell whether aqueous is still flowing along the aqueous veins or whether these vessels are empty. If, however, Evans blue, a non-particulate dye, is introduced into the anterior chamber, it soon appears in the aqueous veins of these animals (Greaves and Perkins, 1951). If the sympathetic is now stimulated, the stream of blue aqueous can be seen to continue flowing even when the other vessels contain little or no blood.

Constriction of the arterioles of the anterior ciliary system following sympathetic stimulation probably causes a lowering of the venous pressure in the collecting vessels, thus allowing an increase in the drainage of aqueous via the aqueous veins.

\section{PART III. Changes in Choroidal Circulation}

(a) INJECTION OF INDIAN INK.-Indian ink was injected into the innominate artery of rabbits while one sympathetic was being stimulated. The animals were killed immediately afterwards, the eyes enucleated and opened, and the choroidal circulation and ciliary region examined under a slit-lamp microscope. The in- 
jected vessels were seen to be contracted on the side of the stimulation; this was most marked in the region of the ciliary body.

(b) Direct Observation of Choroidal Circulation.-During the course of observations on the aqueous veins of rabbits, it was noticed that, unless the eye was kept moist, exposed scleral regions tended to appear black, because an increase in transparency allowed the choroidal pigment to show through.

In albino rabbits with no choroidal pigment, it was thought that the choroidal vessels might be made visible. After some trial experiments, it was found that an excellent view of the choroid could be obtained by the following technique:

In an anaesthetized albino rabbit the conjunctiva is dissected off the area of sclera to be examined. Sutures are inserted in the recti muscles to hold the eye in the desired position. A stream of warm air is now directed on to the bare sclera and in about 5 minutes the area shows a red patch indicating that the sclera is now transparent. A drop of oil on this surface improves the optical conditions, and bright focal illumination through the pupil to illuminate the area of choroid involved allows the choroidal vessels to be examined in detail using the binocular microscope of a Haag-Streit slit lamp.

With this technique the long posterior ciliary arteries can be seen running forward to the root of the iris where they divide to form the major circle of the iris. A much convoluted network of veins can be seen to accompany them (Fig. 3). The vortex veins are easily recognized and the marked constriction during their passage through the sclera is noted. Branches of the short ciliary arteries appear as fine vessels running between the choroidal veins, crossing under and over them. These arterial branches are continued right up to the formation of the vortex vein, and some give the appearance of joining a vein directly. We have not been able to demonstrate any direct arterio-venous connection. On several occasions, by injection of Indian ink into the carotid, an apparent connection has been shown to be an illusion caused by the artery disappearing beneath the vein.

It is certain, however, that the flow from artery to vein is extremely rapid as a substance such as Indian ink injected into the carotid appears in the choroidal veins as soon as it can be seen in the arteries. In such experiments it is interesting to note that with the rectus muscles detached, although the whole circulation of the choroid and iris is easily injected, no injection material appears in the aqueous veins or in the episcleral veins unless a higher pressure is used. This suggests that there is little connection between the two vascular systems within the eye. In confirmation of this, it is found that Evans blue injected under pressure into the anterior chamber rapidly fills the aqueous veins and all the episcleral veins, but does not enter the choroidal circulation. These experiments confirm the investigations of Seidel (1924) and Kiss (1943), who found that indigo, carmine, and Indian ink injected into the anterior chamber filled the ciliary, intrascleral, and episcleral veins, but did not enter the iridic, choroidal, and vortex veins.

It therefore seems likely that the drainage of aqueous from the anterior chamber is confined to the episcleral circulation and that drainage into the choroid, if it occurs at all, is minimal.

\section{Stimulation of Cervical Sympathetic}

If the choroidal circulation is watched by the above method while the sympatnetic nerve in the neck is stimulated, no changes in the visible vessels can be seen. There is a lightening of the background colour between the main vessels, which is presumably due to constriction of the choroidal capillaries. A similar change can be produced by the intravenous injection of adrenaline in doses of 20 to $200 \gamma$. 
If the circulation is slowed down by partial occlusion of the ipsilateral carotid, the flow in the choroidal veins becomes easily visible, and, under these conditions, stimulation of the sympathetic results in a further slowing of the stream.

This effect is not nearly so striking as the changes which occur in the episcleral vessels. It is possible that the drying of the sclera interrupts the flow of nervous impulses in the sympathetic fibres, for it was noted that pupillary dilatation was less marked in the segment of the iris related to the area under observation. In an attempt to obviate such an effect, a lamellar resection of the sclera was done before drying. The thin layer remaining was almost transparent and required very little drying before a good view of the choroid could be obtained. Under these conditions, good pupillary dilatation was obtained on sympathetic stimulation, but the changes in the circulation in the choroid were not appreciably increased.

It therefore seems probable that, apart from some effect on the chorio-capillaris, the sympathetic system does little to control the choroidal blood vessels.

\section{Summary}

(1) Section of the cervical sympathetic nerve had little effect on the intra-ocular pressure in the cat.

(2) Stimulation of the cervical sympathetic nerve in the cat caused an initial rise followed by a fall in intra-ocular pressure. The initial rise is probably due to contraction of smooth muscle in the orbit.

(3) Stimulation of the cervical sympathetic caused an apparent increase in the drainage of aqueous fluid into the aqueous veins of the rabbit.

(4) Stimulation produced some contraction of the chorio-capillaris but no marked changes in the larger choroidal vessels. The vessels were observed in vivo by dehydrating the sclera of albino rabbits. A similar indeterminate result followed the intravenous injection of adrenaline.

\section{REFERENCES}

ADAMÜK, E. (1866). Zbl. med. Wiss., 4, 561

ADLER, F. H. (1924). Arch. Ophthal., N.Y., 53, 1.

ATTREE, V. H. (1950). J. sci. Instrum., 27, 43.

BOURQUIN, E. (1925). Rev. gén. Ophtal., Paris, 39, 491.

Colle, J., Duke-Elder, P. M., and Duke-Elder, S. (1931). J. Physiol., Lond., 71, 1.

Davson, H., and Matchett, P. A. (1951). Ibid., 113, 387.

- and PuRvis, C. (1950). British Journal of Ophthalmology 34, 351.

Graser, E. (1883). Dissertation, Erlangen.

Greaves, D. P., and PerkINS, E. S. (1951). British Journal of Ophthalmology 35, 119.

VON HIPPEL, A., and GRÜNHAGEN, A. (1868). v. Graefes Arch. Ophthal., 14 (part 3), 219.

HöltzKe, H. (1883). Ibid., 29 (part 2), 1.

Henderson, E. E., and Starling, E. H. (1904). J. Physiol., Lond., 31, 305.

JAFFe, N. S. (1948). Amer. J. Ophthal., 31, 1597.

Kiss, F. (1943). Ophthalmologica, Basel, 106, 225.

(1949). Szemészet, 86, 1.

Lagrange, F., and Pachon, V. (1900). C. R. Soc. Biol., Paris, 52, 990.

LEBER, T. (1903). Graefe-Saemisch "Handbuch der gesamten Augenheilkunde", p. 338.

Lodato, G. (1906). Ann. Oculist., Paris, 135, 246.

ROChat, G. F. (1919). Klin. Mbl. Augenheilk., 62, 267.

SCHMERL, E., and STEINBERG, B. (1949). Amer. J. Ophthal., 32, 947.

SeIDEL, E. (1923). v. Graefes. Arch. Ophthal., 112, 252. (1924). Ibid., 114, 157.

SCHMIDT-RIMPLER, H. (1900). Ber. ophthal. Ges. Heidelberg, 28, 29.

STOCK, W. (1910). Klin. Mbl. Augenheilk., 48, 124 (B-H).

THEL, R. (1929). Ibid., 82, 109.

VIDAL, F., and Malbran, J. L. (1944). Ophthal. ibero-amer., 6, 257.

WeEkers, R., and PRIJOT, E. (1950). Ophthalmologica, Basel, 119, 321.

Wessely, K. (1908). Arch. Augenheilk., 60, 1.

YATA S. (1930). Acta. Soc. ophthal. jap., 34 (Festschr. Ichikawa), 550 [in Japanese]. 